\title{
Detecting Coherent Structures in a Turbulent Wake by Using Delay Based Networks
}

\author{
F. López Peña, R. J. Duro, and M. Sánchez Simón. \\ Universidad de la Coruña, Escuela Politécnica Superior, Mendizabal s.n., E-15403 \\ Ferrol, Spain.
}

\begin{abstract}
A delay based artificial neural network is used to analyze turbulent flow signals obtained by a hot wire anemometer along time in different points within the wake of a circular cylinder. Values of the Reynolds number range from 2000 to 8000 , corresponding to late transitional or fully turbulent wake flows. The implemented trainable delay based artificial neural network is able to autonomously obtain the embedding dimension as well as the normalized embedding delay and permits performing short and long term predictions. The short term predictions are extremely accurate while the long term ones result in a sort of non linear filter able to extract the signal features concerned with the larger eddies and coherent structures present in the turbulent flow-field.
\end{abstract}

Keywords: Turbulence, Turbulent Wake, Von Karman Vortex Street, Coherent Structures, Artificial Neural Network, Hot Wire Anemometer.

\section{Introduction}

Turbulence in flows can be somehow referred to as a spatial-temporal chaos with a large number of degrees of freedom resulting in a wide range of scales. Being a deterministic phenomenon the classical approach was statistical Only during the last two or three decades some other approaches have been taken. It is important to point out that the dynamics and the transfer processes of momentum, heat and mass in most turbulent flows of interest are governed by the larger eddies and coherent structures embedded in these turbulent flows, while the smallest scales of turbulence are responsible for dissipation. Coherent structures usually appear at the transition stages of turbulence and remain present in the flow far downstream when the fully developed small isotropic scales of turbulence dominate the flow field. However, as is the case for other large scales of turbulence, they cannot be considered as a part of the fully developed turbulence. These facts are the bases of large-eddy simulation (LES) which is a promising approach for computing turbulent flows. In the LES approach only the evolution of the large scales is computed while the small scales of turbulence are modeled and filtered out after considering them almost isotropic. Also, in experimental and practical applications, the analysis, identification and monitoring of the larger structures can be used to control turbulence. [1]

In our study, a hot wire anemometer is used to measure velocities in the turbulent wake of a cylinder. For many years, the hot wire anemometer has been a basic tool for experimental characterization of turbulent flows. The main characteristic that makes this device very suitable for turbulence measurements is its ability to measure flow fluctuations up to the hundred thousand $\mathrm{Hz}$ range. Notwithstanding the dramatic advances achieved during the last decades in developing new experimental flow measurement and diagnostic apparatus, none of these are able to beat the frequency response of the hot wire anemometer [2].

The flow around a circular cylinder and the wake behind, have been extensively studied in the past. Recently it is been used as a work bench for experimental and numerical studies. The wake presents many different modes and regimes governed by a single control parameter; the Reynolds number, which is defined as de ratio between inertial and viscous forces. For a cylinder of diameter $D$ moving at velocity $V$ in a fluid of density $\rho$ and viscosity $\mu$, the Reynolds number has a value $\operatorname{Re}=\rho V D / \mu$. Some of the regimes characterizing this flow are represented in Figure 1 . At Reynolds numbers higher than 35 the wake starts to oscillate and soon after begins forming pairs of vortices shed alternately from each side of the rear surface of the cylinder; this is called the Karman vortex street. As the Reynolds number rises, the wake becomes more complex and turbulent, but still the vortices of the Karman vortex street can be detected in the form of coherent structures set in the turbulent wake up to very high values of the Reynolds number. It is important to notice that for $\mathrm{Re}<200$ the flow is basically laminar and the Karman vortex street persists very far downstream of the cylinder, but for higher values of the Reynolds number the wake becomes turbulent, the Karman street starts to degenerate into a turbulent wake and the vortices, interacting in a complex manner, become unstable and start to split into smaller ones vanishing after a short path downstream. In any case de cylinder can be seen as a source of perturbation in the unperturbed flow-field. The higher the Reynolds number, the higher the turbulence becomes and, consequently, the length where the Karman street can be detected becomes shorter. Very complicated three dimensional patterns appear in the flow-field during the transition from a well organized, periodic and two-dimensional Karman street to a fully turbulent wake. The visualization and analysis of such patterns has been the subject of several experimental and numerical studies as, for instance, in [3] and [4]. 

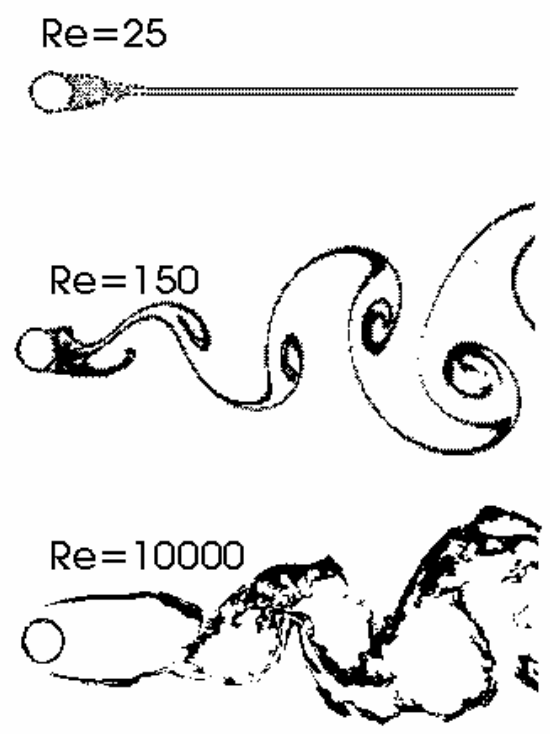

Figure 1.- The wake of a circular cylinder at three ditterent flow regimes.

The dimensionless cylinder shedding frequency is called the Strouhal number; $S t=f D / V$. The variation of the Strouhal number with the Reynolds number in the wake of a circular cylinder for a laminar incoming flow is represented in Figure 2 using data coming from many sources. Data is taken in the vicinity of the cylinder and spread increases with distance or if turbulence in the incoming flow does. For Reynolds numbers from $2 \cdot 10^{2}$ to $10^{5}$, the Strouhal number appears to be approximately equal to 0.2 . As a consequence, after the Strouhal number definition it can be deduced that the distance between two consecutive vortices in the Karman street along the main flow direction is about 5 cylinder diameters. [5]

Particularizing to the application of this paper, an artificial neural network is the means for the identification of the coherent structures within the turbulent wake. The analysis is made on the signal taken along a period of time in a given spatial position by a hot wire anemometer. In the realm of signal identification and modeling, the main problem is to obtain a model of the signal that is as accurate as possible and which permits performing short and long term predictions. This is, we are interested in the dynamic reconstruction of the measured signal and we are mainly concerned with the larger eddies and coherent structures present in the turbulent flow-field, which are related to signal fluctuations with larger characteristic time variation.

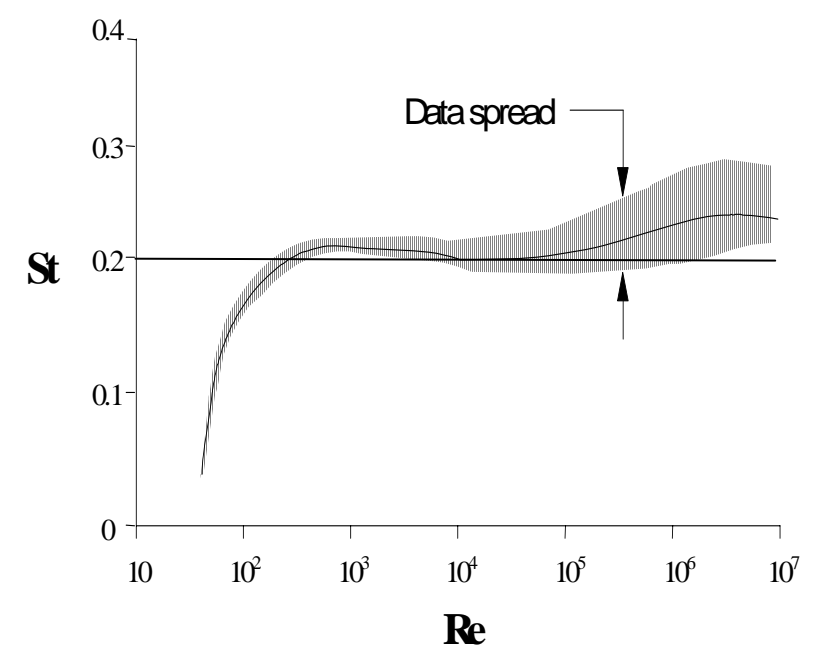

Figure 2.- Strouhal number versus Reynolds number in the wake of a circular cylinder.

In the literature one can find different methods to identify coherent structures in turbulence, such as flow visualization, spectral and correlation analysis, principal component analysis or proper orthogonal decomposition, conditional sampling and pattern recognition among others. Despite their potentiality, the use of artificial intelligence and neural networks for this purpose is rare. One of these few papers that are somehow related to the scope of the present work, is the paper by Fere-Gine et al. [6]. They use a Fuzzy Artmap Neural Network to analyze the coherent structures embedded in two-component velocity signals measured simultaneously by an array of five hot wire probes in a turbulent wake behind a circular cylinder. 
Usually, most neural network based implementations have not taken into account the basic premises on which the modeling of signals must be based, that is, that in order to model a time dependent non linear signal one must be able to define its state space, or an equivalent state space, in an unambiguous manner in order to prevent multivalued sets of coordinates which would lead to ambiguous predictions. In fact, one of the best areas to appreciate this is in the case of chaotic time series, where if the state space is not well chosen the orbits of the series in this space will cross, generating points of ambiguity. In this article we make use of a trainable delay based artificial neural network that directly implements a form of the embedding theorem [7] [8] with the advantage of being able to autonomously obtain the embedding dimension and the normalized embedding delay.

As a first step in directly training the delays introduced in a generalized synaptic delay network using a gradient descent method, we have developed the DTB algorithm [9]. It leads to easy and precise training of temporal event processing networks straight from the input signals, with no windowing or preprocessing required and, what is more important, the embedding dimension does not have to be defined a priori. In the context of signal processing, and taking into account that many signal related correlations take place in the frequency domain, we have now added the possibility of including product terms in some of the nodes which, combined with the automatic selection of the signal points to be multiplied (through the training of the delay terms), allow for learnable signal correlation operations within the network.

\section{EXPERIMENTAL SET-UP}

The experiments were carried out in our Low Turbulence Subsonic Open Jet Wind Tunnel (LTOJ-1) sketched in Fig. 3. This is a low speed low turbulence wind tunnel specially designed and built in house for anemometer calibration and for basic research purposes. The tunnel produces a $300 \mathrm{~mm}$ wide open free jet with a velocity uniformity of better than $1 \%$ and turbulence level of less than $1 \%$. It is driven by a centrifugal blower provided with an $11 \mathrm{~kW}$ asynchronous AC motor. An electronic invertor is used for controlling the motor speed and provides a simple continuously variable air speed control from 0 to $45 \mathrm{~m} / \mathrm{s}$. This invertor produces a variable frequency power supply to the AC motor and is controlled by a DC signal from 0 to 10 volts proportional to the output frequency.

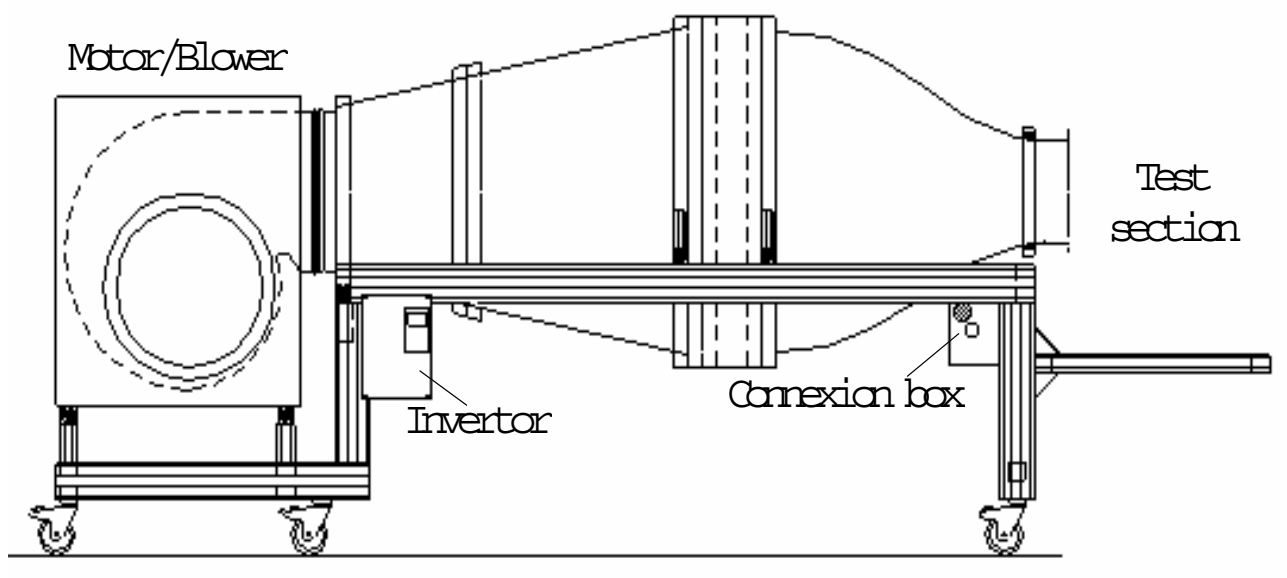

Figure 3.- The LTOJ-1 wind tunnel.

A software package constituting a virtual instrument was specially developed in house to perform data acquisition and control of both the hot wire anemometer processor trough a RS232 line and the wind tunnel through an output channel of the acquisition board connected to the invertor control line. The virtual instrument is a new version of the one presented in [10]. A set of sensors are arranged to measure the different physical magnitudes needed within this virtual instrument to control the wind tunnel motor by means of a PID controller embedded in the software package. Thus, a differential pressure transducer connected to a Pitot-static Prandtl type pressure probe allows the measurement of the main airflow dynamic pressure $P d$. To obtain the wind speed from this magnitude we must know the density of air under the test conditions, for this purpose a barometric pressure sensor and a temperature sensor are used. Air density is calculated from the estate equation as $\rho=P a / R g T a$, being $R g$ the air constant and $P a$ and $T a$ the ambient pressure and temperature respectively. Data from an electronic hygrometer are also acquired and used to correct the air density value for relative humidity effects. Then the air velocity is calculated by using the Bernouilli equation as $V=\sqrt{ }(2 P d / \rho)$. All the mentioned sensors, as well as the hot wire anemometer output signal and the AC motor invertor control line, are connected to the data acquisition board installed in a personal computer trough a connection box where power supplies and filters for the sensors are installed. The experimental set-up is schematically represented in Fig. 4. 


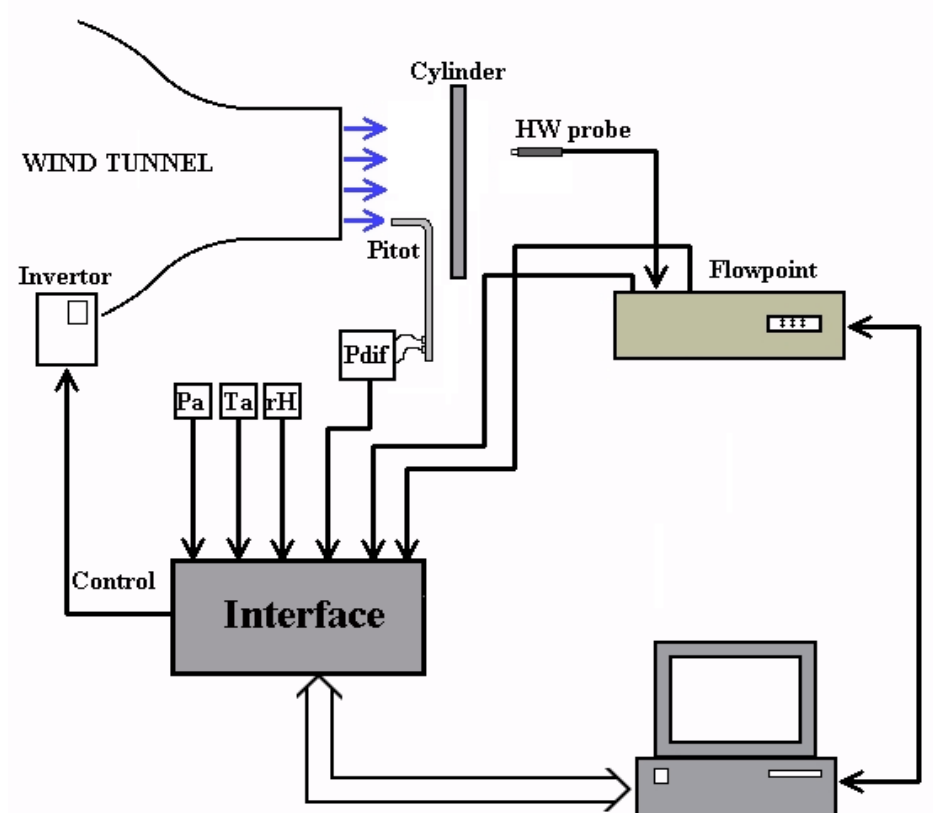

Figure 4.- The experimental set-up.

A circular cylinder with a diameter of $8 \mathrm{~mm}$ is placed vertically in the test section at the open jet exit. The Reynolds number is used as the main control parameter. According to the expression given below, this number depends on the cylinder diameter and on the air velocity, density and viscosity. The cylinder diameter is passed to the software package as a parameter, the air density and velocity had been already calculated and the air viscosity is obtained from the measured ambient temperature by using a simplified correlation. The software package has been developed to be able to calculate the Reynolds number in real time and to adjust the wind tunnel speed through the embedded PID controller to stabilize its value within $1 \%$.

Velocity measurements are taken by means of a commercial TSI Flowpoint hot wire anemometer at positions placed 10, 20 and 40 diameters downstream of the cylinder, these distances correspond, respectively, to 2, 4 and 8 wave lengths of the von Karman vortex street. In the first position, the characteristic frequency should be distinguishable by traditional means, e.g. Fourier transform, up to Reynolds numbers higher than $10^{6}$, as shown in Figure 2. In the second position the increased turbulence downstream makes the corresponding spectrum peak diminish until it vanishes for Re in the $10^{3}$ range. In the third position the vanishing of the power spectrum peak occurs at Re values of only a few hundreds.

We considered values for this parameter ranging from 2000 to 8000, corresponding to a late transitional or almost fully turbulent wake with a von Karman vortex street still present. As we are interested in extracting the main signal features under extremely noisy conditions, the main flow turbulence level was forced up to $2 \%$ during the tests, so as to increase the spread shown in figure 1 and to increase complexity in the turbulent signal making it more difficult to analyze by linear means. Nevertheless, as the measurements are taken in the near wake, large eddies and coherent structures are present, resulting in strong fluctuations of the measured air flow velocities.

\section{DYNAMIC RECONSTRUCTION}

The problem usually referred to as Dynamic Reconstruction involves obtaining some type of description of a given chaotic time series obviating the need for detailed mathematical knowledge of the underlying processes that conform its dynamics.

It is well known that the state of any dynamical system in a given instant of time may be specified by means of a state space vector. The coordinates of this state space vector are independent degrees of freedom of the system. In particular if, as in the case of Haykin and Principe [11], we consider a system described by a non linear difference equation:

$x(n+1)=F(x(n))$

where $x(n)$ is the d-dimensional state vector of the system in time $n$ and $F()$ a vector valued function. And we assume that:

$y(n)=h(x(n))+w(a)$

is the time series measured at the output of the system in terms of $x(n)$, where $h()$ is a scalar valued function and $w(n)$ represents additive noise that takes into account the combined effects of imperfections and imprecision in the 
observable. That is, $y(n)$ is what we can measure and what we want to obtain a model for without having to resort to a precise description of the underlying mechanisms leading to it.

The Embedding theorem [7] [8] states that the geometric structure of the mutivariable dynamics of the system can be unfolded from observable $y(n)$ with $w(n)=0$ in a D-dimensional space constructed from the new vector:

$y_{R}(n)=[y(n), y(n-\tau), \ldots, y(n-(D-1) \tau)]^{T}$

where $\tau$ is the Normalized Embedding Delay usually taken as a positive integer, even though sometimes it may be useful for it to be a positive non integer.

From this theorem, it is established that when there is a single measured quantity for a dynamic system, it is possible to reconstruct a state space that is equivalent to the unknown original space composed of all the dynamical variables using the D-dimensional vector $y_{R}(n)$ if $D>=2 d+1$ being $d$ the dimension of the state space of the system. $D$ is called the embedding dimension.

Basically this implies that if the system produces orbits in the original state space that lie on a geometric object of dimension d (not necessarily an integer), we can avoid having spurious intersections of the orbits, which would obviously result in points where there would be two or more possible next values, that is an ambiguity in the function, by seeing the object in another space of integer dimension $D$. The coordinates in this second space are nonlinear transformations of the original state-space coordinates.

The main problem with this approach is that we require reliable estimates of the embedding dimension which are not simple to calculate, although several authors describe some methods for obtaining it [12] [13]. Another problem is the fact that the theorem has nothing to say on the choice of the Normalized Embedding Delay. In practice, $\tau$ is taken so that it is large enough for $y(n)$ and $y(n-\tau)$ to be independent of each other and thus be coordinates of the reconstruction space, but not so independent as to have no correlation with each other. Some authors suggest that the best choice would be a $\tau$ where the mutual information for $y(n)$ and $y(n-\tau)$ attains its first minimum [12] [14].

It is clear that the strategies for obtaining these parameters are sort of "rule of the thumb". Given the fact that they are critical for an appropriate reconstruction of the signals, in what follows we have decided to include these two parameters in the architecture of the network. Consequently, during the learning process both, the Normalized Embedding Delay and the Embedding Dimension will be autonomously selected. The former indirectly through the choice of what instants of time are considered for the reconstruction of the signal and the latter through the choice of how many of these instants are selected.

\section{Discrete Time Backpropagation with П Units}

The artificial neural network we consider for training consists of several layers of neurons connected as a Multiple Layer Perceptron (MLP), it is represented schematically in Fig. 5. There are two differences with respect to traditional MLPs. The first one is that the synapses include a delay term in addition to the classical weight term. That is, now the synaptic connections between neurons are described by a pair of values, $(\mathrm{W}, \tau)$, where $\mathrm{W}$ is the weight, representing the ability of the synapse to transmit information, and $\tau$ is a delay, which in a certain sense provides an indication of the length of the synapses. The longer it is it will take more time for information to traverse it and reach the target neuron. The second one is that some of the nodes implement a product combination function instead of the traditional sum.

We have developed an extension of the backpropagation algorithm for training both parameters of the connections, and have called it Pi Discrete Time Backpropagation (П-DTB) [15]. This algorithm permits training the network through variations of synaptic delays and weights, in effect changing the length of the synapses and their transmission capacity in order to adapt to the problem in hand.

In addition, through the appropriate determination of the delay terms in the synapses the П-DTB algorithm performs an automatic selection of the signal points to be correlated. Consequently, when speaking in the language of the dynamic reconstruction of signals, the network automatically obtains the embedded delay and embedded dimension.

If we take into account the description of the network in terms of synaptic weights and synaptic delays, the main assumption during training is that each neuron in a given layer can choose which of the previous outputs of the neurons in the previous layer it wishes to input in a given instant of time. Time is discretized into instants, each one of which corresponds to the period of time between an input to the network and the next input. During this instant of time, each of the neurons of the network computes an output, working its way from the first to the last layer. Thus, for each input, there is an output assigned to it.

In order to choose from the possible inputs to a neuron the ones we are actually going to input in a given instant of time, we add a selection function to the processing of the neuron. This selection function could be something as simple as:

$\delta_{i j}=\left\{\begin{array}{l}1 \rightarrow i=j \\ 0 \rightarrow i \neq j\end{array}\right.$ 


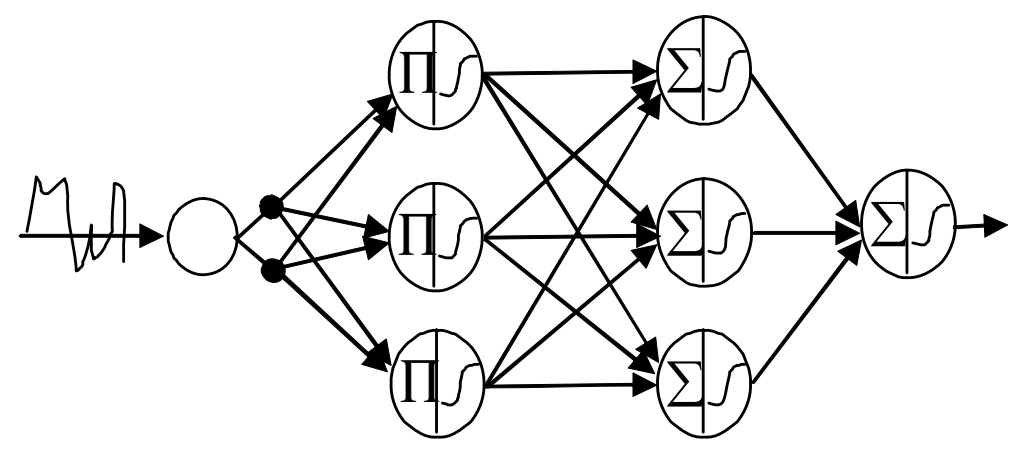

Each synapse consists of a trainable weight and delay term

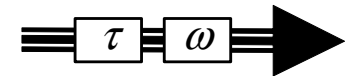

Figure 5 - Network used.

so that the output of a traditional (5) and a product (6) node $k$ in an instant of time $t$ is given by:

$$
\begin{aligned}
& O_{k t}=F\left(\sum_{i=0}^{N} \sum_{j=0}^{t} \delta_{j\left(t-\tau_{i k}\right)} w_{i k} h_{i j}\right) \\
& O_{k t}=F\left(\prod_{i=0}^{N} \sum_{j=0}^{t} \delta_{j\left(t-\tau_{i k}\right)} w_{i k} h_{i j}\right)
\end{aligned}
$$

where $F$ is the activation function of the neuron, $h_{i j}$ is the output of neuron $i$ of the previous layer in instant $j$ and $w_{i k}$ is the weight of the synapse between neuron $i$ and neuron $k$. The first sum (or product) is over all the neurons that reach neuron $k$ (those of the previous layer) and the second one is over all the instants of time we are considering.

The result of this function is the sum or product of the outputs of the hidden neurons in times $t-\tau_{i k}$ (where $\tau_{i k}$ is the delay in the corresponding connection) weighed by the corresponding weight values.

Now that we know what the output of each neuron is as a function of the outputs of the neurons in the previous layer and the weights and delays in the synapses that connect these neurons to it, what we need is an algorithm that allows us to modify these weights and delays so that the network may learn to associate a set of inputs to a set of outputs. The basic gradient descent algorithm employed in traditional backpropagation may be used, but we must now take into account the delay terms when computing the gradients of the error with respect to weights and delays.

As shown in [9], these gradient terms are:

$$
\begin{aligned}
& \frac{\partial E_{t o t a l}}{\partial w_{j k}}=\Delta_{k} h_{j\left(t-\tau_{j k}\right)} \\
& \frac{\partial E_{t o t a l}}{\partial \tau_{j k}}=\Delta_{k} w_{j k}\left(h_{j\left(t-\tau_{j k}\right)}-h_{j\left(t-\tau_{j k}-1\right)}\right)
\end{aligned}
$$

being $E_{\text {total }}$ the total squared error for all the training vectors and

$$
\Delta_{k}=\frac{\partial E_{\text {total }}}{\partial O_{k}} \frac{\partial O_{k}}{\partial \text { ONet }_{k}}=2\left(O_{k}-T_{k}\right) F^{\prime}\left(\text { ONet }_{k}\right)
$$

where $T_{k}$ is the desired output, $O_{k}$ the one really obtained and $O_{N e t k}$ is the combination of inputs to neuron $k$, when we consider output neurons. For hidden neurons connected to the input layer, and defining as before

$\Delta_{k}=\frac{\partial E_{\text {total }}}{\partial h N e t_{k}}=F^{\prime}\left(h N e t_{k}\right) \sum_{r} \Delta_{r} w_{k r}$ 
where index $r$ represents the neuron of the next layer, whether output or hidden, we have the following derivatives for the weights and connections:

$$
\begin{aligned}
& \frac{\partial E_{\text {total }}}{\partial w_{j k}}=\frac{\partial E_{\text {total }}}{\partial h N e t_{k}} \frac{\partial h N e t_{k}}{\partial w_{j k}}=\Delta_{k} I_{j\left(t-\tau_{j k}\right)} \\
& \frac{\partial E_{\text {total }}}{\partial \tau_{j k}}=\frac{\partial E_{\text {total }}}{\partial h N e t_{k}} \frac{\partial h N e t_{k}}{\partial \tau_{j k}}=\Delta_{k} w_{j k}\left(I_{j\left(t-\tau_{j k}\right)}-I_{j\left(t-\tau_{j k}-1\right)}\right)
\end{aligned}
$$

where the second derivative in (9) is the result of:

$$
\frac{\partial h N e t_{k}}{\partial \tau_{j k}}=\frac{\partial\left[\sum_{i=0}^{N} \sum_{n=0}^{t} \delta_{n\left(t-\tau_{i k}\right)} w_{i k} I_{i n}\right]}{\partial \tau_{j k}}
$$

when we consider neurons in a hidden layer. It may be observed that the derivative in $h N e t$ of equation (13) has been discretized in order to obtain (12), implicitly assuming there is certain continuity in the temporal variation of the outputs of the neurons, which in practice turns out to be a valid assumption.

Regarding the product units in the first hidden layer, we have:

$$
h N e t_{k}=\prod_{r=0}^{N} w_{r k} I_{r\left(t-\tau_{r k}\right)}
$$

and the derivatives are:

$$
\begin{aligned}
\frac{\partial h N e t_{k}}{\partial w_{j k}}=\frac{\prod_{r=0}^{N} w_{r k} I_{r\left(t-\tau_{r k}\right)}}{w_{j k}} \\
\frac{\partial h N e t_{k}}{\partial \tau_{j k}}=\frac{\prod_{r=0}^{N} w_{r k} I_{r\left(t-\tau_{r k}\right)}}{I_{j\left(t-\tau_{j k}\right)}}\left(I_{j\left(t-\tau_{j k}\right)}-I_{j\left(t-\tau_{j k}-1\right)}\right)
\end{aligned}
$$

where index $r$ denotes the input nodes connected to the corresponding product hidden unit. The importance of the procedure is that now the appropriate delays for these connections, and consequently the temporal values that make up the product terms in the pi units are obtained automatically, and are not imposed beforehand.

As we show, by discretizing the time derivative we obtain simple expressions for the modification of the weights and delays of the synapses, in an algorithm that is basically a back-propagation algorithm where we have modified the transfer function of the neuron to permit a choice of inputs between all of the previous outputs of the neurons of the previous layer.

\section{RESULTS}

In what follows we will present some results we have obtained from turbulent signals measured with a hot wire anemometer in the wake of a round cylinder, and which will indicate the capabilities of these networks to grasp the underlying structure of the signal and thus obtain very good prediction results, even when performing multi-step predictions under noisy environments. For clarity we have selected to present here a reduced number of test cases, enough to be representative of the different situations analyzed. This way, all the cases presented save for one were measured under ambient conditions with free air speed just about $7.6 \mathrm{~m} / \mathrm{s}$, matching a Reynolds number of 4000 . Hot wire signals are taken at three positions placed respectively at distances of 10, 20 and 40 diameters downstream of the cylinder, which correspond to 2, 4 and 8 wave lengths from the perturbation source. Figure 6 represents the power spectra of these signals once their amplitudes have been normalized. In the first of these spectra (6.a) we can still see a peak at $200 \mathrm{~Hz}$ corresponding to $\mathrm{St}=0.2$, but for the other two signals (6.b and 6.c) no significant peaks appear. This is quite meaningful according to the underlying physics, because near the perturbation source the coherent structures are strong enough to show their periodic characteristic, but the growing of turbulence downstream wipes this characteristic from the other two signals or, at least, it appears to be undetectable by traditional linear means. 

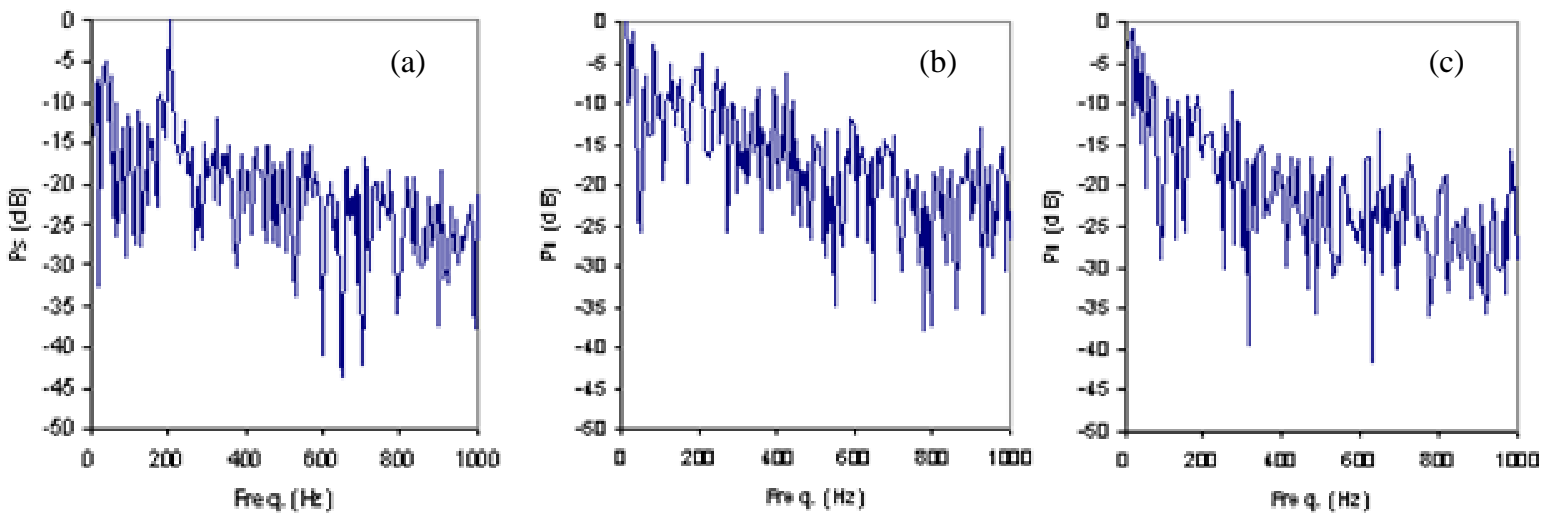

Figure 6.- Power spectra of three hot wire signals taken at 10, 20 and 40 diameters downstream in the wake of a round cylinder with $\mathrm{Re}=\mathbf{4 0 0 0}$.

As a first application, neural networks have been applied to predict the measured hot wire signals. Figure 7 displays the prediction obtained by the networks for the three above mentioned cases. The neural networks employed consisted of a single input (replicated into two inputs to make use of the product terms in the first layer), two hidden layers with 20 or 30 neurons and a single output which provided the predictions different lengths of time into the future. For each case, every signal was treated separately and fed into its corresponding network through its single input, there was no previous windowing process and the networks were trained for 1000 to 3000 epochs on the real signal. The complexity of the signal increases from the first to the third case and, as a consequence, to model it precisely requires more
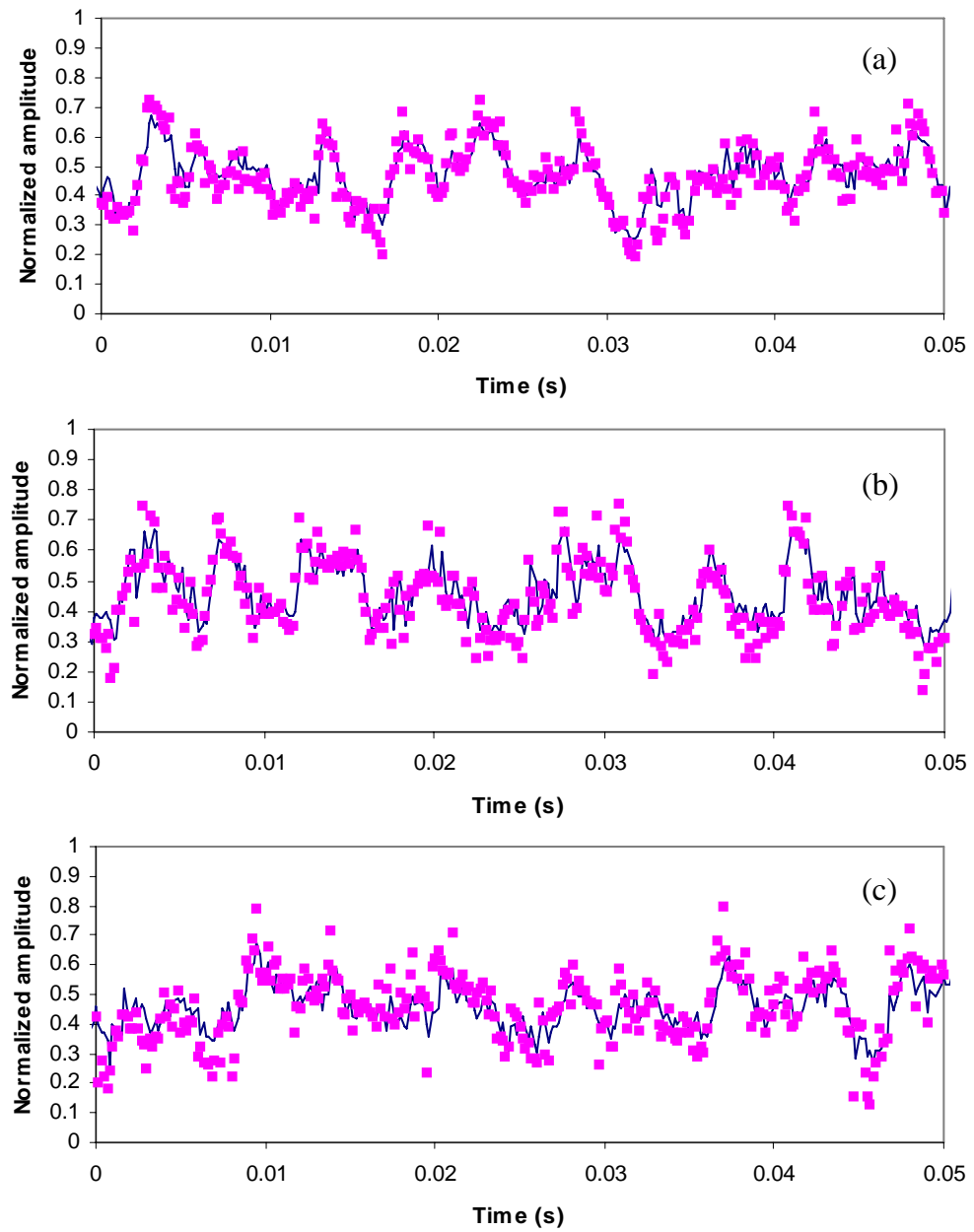

Figure 7 - Real signals (squares) and predictions (line) obtained by a 1-20-20-1 П-DTB trained delay based network in the case of the top graph and 1-30-30-1 networks for the other two. The signals correspond to the spectra of figure $6(\operatorname{Re}=4000)$. 

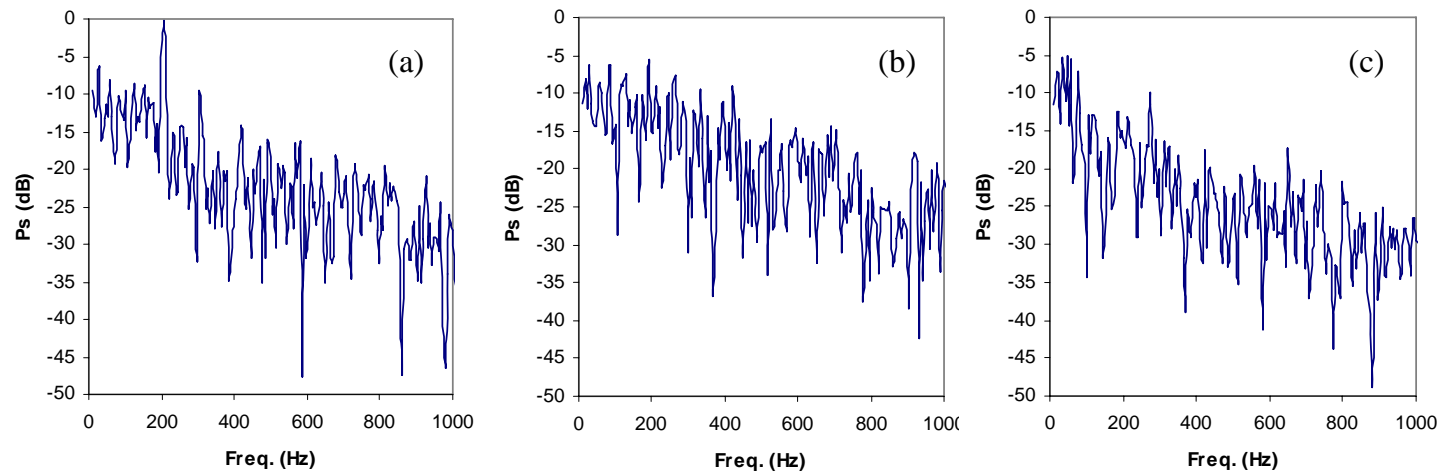

Figure 8.- Power spectra of the signals predicted by neural networks represented in Figure 7.

complex networks leading to longer training times. In fact, the first spectrum only required 20 neurons in the hidden layers while the other two required 30 to obtain the same level of accuracy. As shown the figure, the predictions which the networks learn to perform are quite good; in fact, the MSE for these predictions are around $1.5 \times 10^{-3}$. The power spectra of the predicted signals are displayed in Figure 8, they present the same characteristics as the real signals.

As a second type of experiment, we trained the networks on the real signal and then used them as signal generators. In this case no signal input was provided to the networks from a certain point on and they were allowed to run in parallel
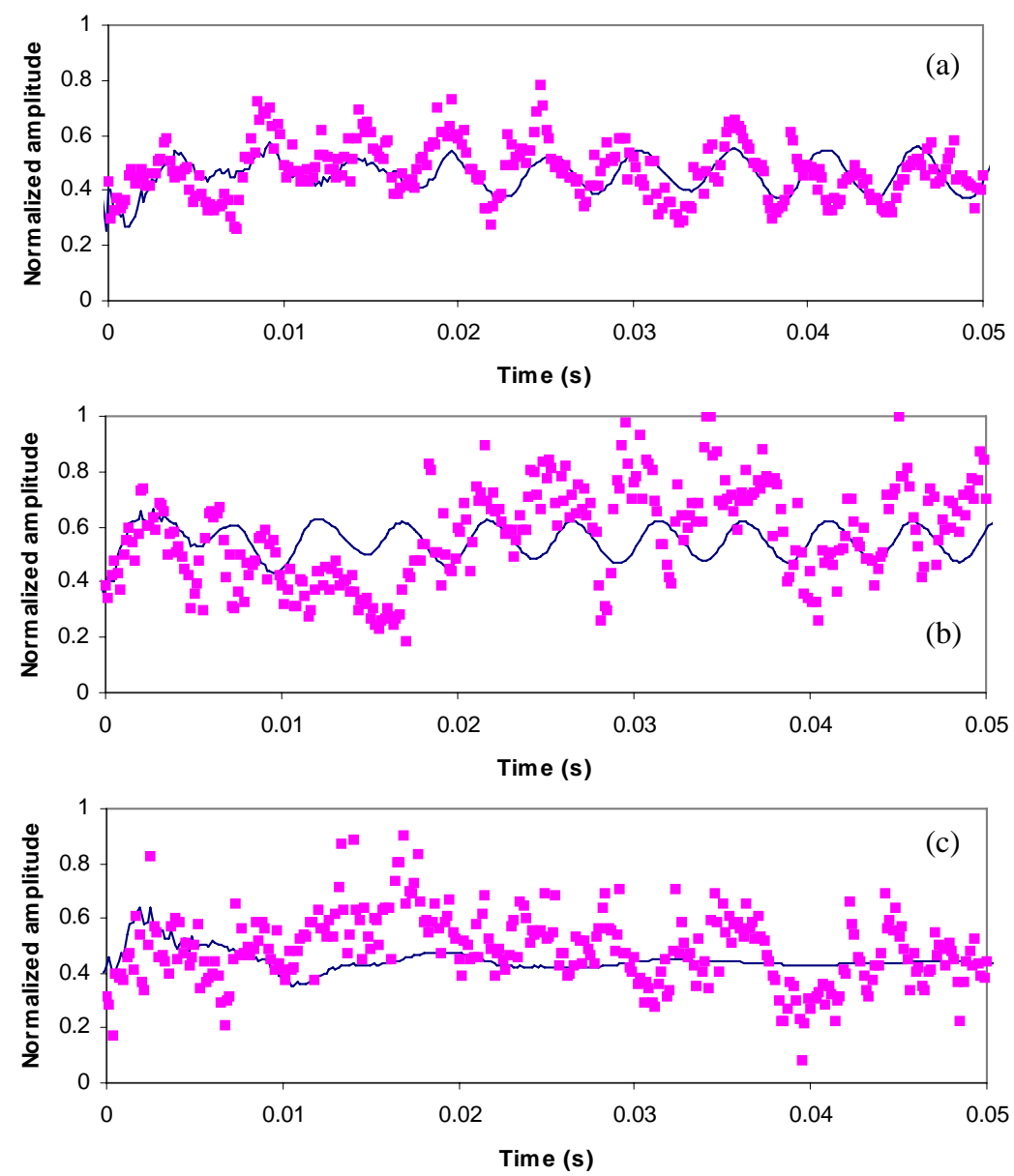

Figure 9. - Real signal (squares) and signal generated (line) by a 1-20-20-1 П-DTB trained delay based network operating as a multistep predictor using its own outputs as inputs for the next prediction. The signals are taken at 10, 20 and 40 diameters downstream in the wake of a round cylinder with $\mathbf{R e}=4000$. 

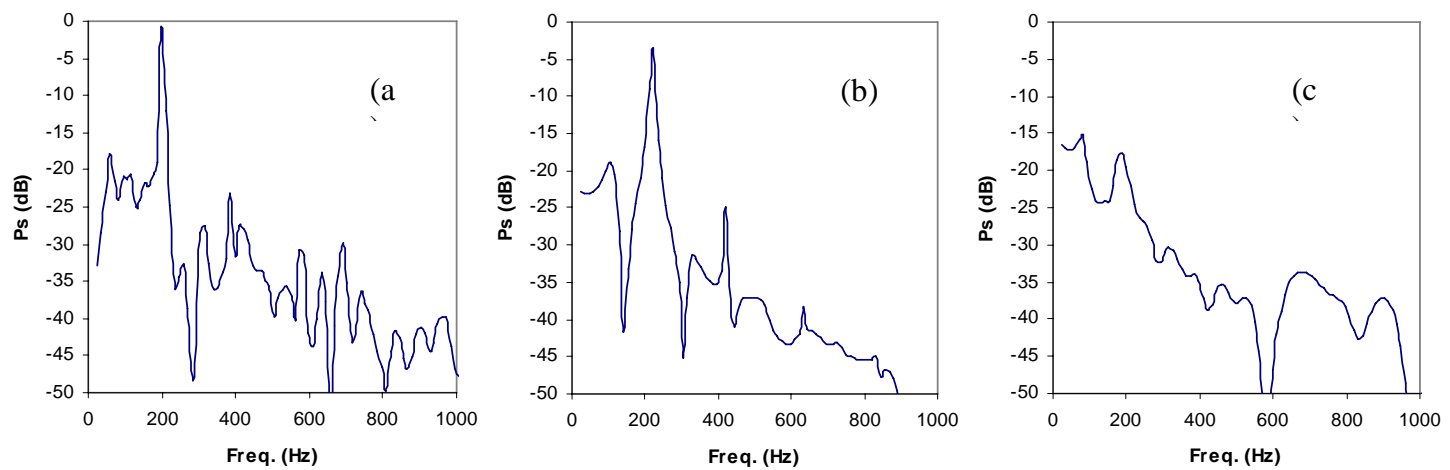

Figure 10.- Power spectra of the signals generated by neural networks represented in Figure 9.

with the signal using their previous outputs as inputs for the next predictions. That is, they were run as multistep predictors or signal generators. This was done in order to see how well they managed to capture the basic dynamics of the process. It must be pointed out again that the main objective of this work is to be able to capture the basic dynamic structure of the process underlying the signals independently from the noise they show and from the higher frequency components induced by perturbing phenomena and by the smaller scales of turbulence. Consequently, the objective is to obtain a repeatable dynamic pattern that is similar enough to the one underlying the signal so that we can make long term predictions on our phenomena. In other words, we expect that the trained neural network should generate a signal with the same features as the coherent structure present in the flow; therefore, the neural network should act as a non linear filter able to extract the main features of the coherent structures out of the rest of the complex signal. In the case of a cylinder wake, the coherent structures are periodic in their origin, so we expect that traces of this periodicity will be present where traditional signal analysis tools are not able to detect them. In the case these assumptions are correct; the power spectrum of the generated signal must have a peak at $200 \mathrm{~Hz}$ corresponding to a Strouhal number of 0.2 .

Figure 9 shows the results of this experiment for the same networks used in the prediction cases but now running in their signal generation mode, that is with no real input. For the first position, 10 diameters downstream of the cylinder, after a short while, the generated signal becomes periodic; this is also the case for the signal taken 20 diameters downstream. However, for the position 40 diameters downstream, a periodic signal is generated but it is damped rather quickly. It is very interesting to observe how the power spectra of all these generated signals, represented in Figure 10 , present the expected peak about $200 \mathrm{~Hz}$. This means that the artificial neural networks have been able to extract out of the chaotic signal the information concerning the periodic generation of the coherent structures which appears not to have vanished completely in the turbulent wake. For the case where the measurements were taken further downstream, a second peak grows about $80 \mathrm{~Hz}$, this means that for this position a second main oscillation appears in the signal.
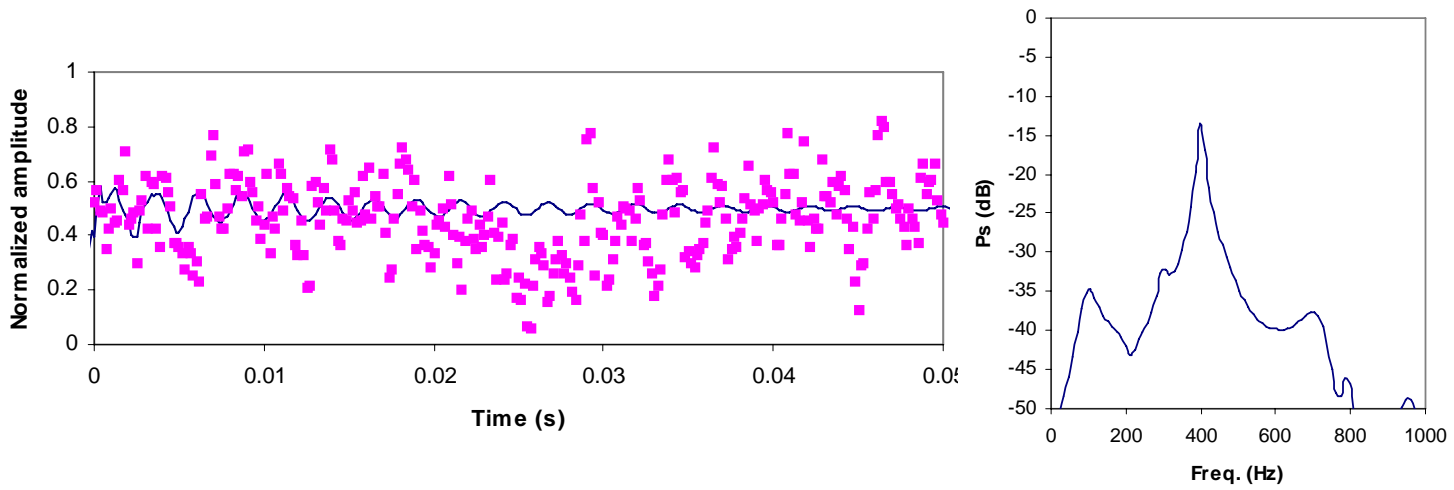

Figure 11. - Left: Real signal (squares) and signal generated (line) by a 1-20-20-1 П-DTB trained delay based network operating as a multistep predictor using its own outputs as inputs for the next prediction. Right: Power spectrum of the generated signal. Data taken 10 diameters downstream of a cylinder with $\mathbf{R e}=\mathbf{8 0 0 0}$.

There are some significant remarks to be made. For the first signal, taken 10 diameters downstream, the power spectrum analysis is able to show that an important $200 \mathrm{~Hz}$ component is present in the wake. In this case, after a short transition where less remarkable features of the signal are progressively lost, the artificial neural network starts to generate a periodic signal corresponding to that frequency. For the second signal, taken 20 diameters downstream, the power spectrum analysis is not able to detect any dominant frequency. On the other hand artificial neural networks can generate for this case a periodic signal, as shown in Figure 10, but this will only happen if the network has been carefully dimensioned and set-up, in any other case a damped periodic signal such as the one shown for the case 40 diameters downstream will be produced. Anyway, even when the generated signal is damped, it oscillates at the correct frequency, therefore the right flow characteristic is being extracted. This has also been the case for different Reynolds numbers between 2000 and 8000 used in the experiments. As an example, Figure 11 shows the generated signal and the corresponding power spectrum of a test case corresponding to a $\mathrm{Re}=8000$, with air speed of $15 \mathrm{~m} / \mathrm{s}$ and frequency of 
$400 \mathrm{~Hz}$ equivalent to $\mathrm{St}=0.2$. From this spectrum we can see that the generated signal has a strong peak at the correct main frequency.

\section{CONCLUSIONS}

In this paper we have considered the problem of modeling and predicting the signals obtained from hot wire anemometer measurements by means of artificial neural networks that intrinsically contemplated the dynamical aspects of the model. That is, we have approached the signal reconstruction problem through the introduction of trainable delay terms in the synapses of the networks. These networks do not require any type of windowing and can be trained to learn the required embedding dimension and normalized embedding delay by the simple application of the signal through a single input as time progresses. To train these networks we have made use of the Pi-Discrete Time Backpropagation algorithm which considers delays in every synapse and, in addition, considers product terms in the first layer.

The application we present corresponds to the prediction of the signal obtained by a hot wire anemometer in the turbulent wake of a round cylinder. These signals can become quite chaotic depending on the downstream position in the wake where the signals are measured and on the Reynolds numbers. We have chosen the test conditions to mach Reynolds numbers between 4000 and 8000 . Hot wire anemometer data were taken at three positions located 10, 20 and 40 diameters downstream of the cylinder. These positions correspond to the near wake, in the case of the first one, and to the fully developed wake in the case of the last one.

The results show that the application of delay based networks is a very promising approach. The predictions obtained by the networks are quite good, and if we operate in a signal generation mode, that is, the network makes predictions using as inputs its previous predictions so that a new signal is generated, the resulting signal starts being quite similar to the real one, and then it degenerates to a periodic signal or a damped periodic signal. In all the cases tested, the main frequency of the generated periodic signal, damped or not, corresponds to the main periodic characteristic of the major coherent structures present in the turbulent signal. This is quite remarkable because for all present test cases, excluding the one of $\operatorname{Re}=4000$ at 10 diameters downstream from the cylinder, traditional linear analysis tools are not able to extract this main frequency. Therefore, the use of non linear tools reveals that the periodic characteristic of the coherent structures in a turbulent wake persist a long way downstream from the point were traditional analysis tools are no longer able to detect it.

\section{ACKNOWLEDGMENTS}

This work was partially funded by FEDER through project N. 1FD97-1700 and by the Spanish MCYT trough project TIC2000-0739-C04-04.

\section{References}

[1] M. Lesieur. Turbulence in Fluids, Third Revised and Enlarged Edition. Kluwer Academic Publishers. Dordrecht, 1997.

[2] H. H. Bruun, Hot-wire anemometry: principles and signal analysis. Oxford University Press. Oxford, 1995.

[3] M. Brede, H. Eckelmann and D. Rockwell, On secondary vortices in the cylinder wake, Phys. Fluids, vol 8, pp 2117-2124, 1996.

[4] M. C. Thompson, K. Hourigan and J. Sheridan, Three-dimensional instabilities in the wake of a circular cylinder, Experimental and Thermal Fluid Science, vol 12, pp 190-196, 1996.

[5] F.M. White, Viscous Fluid Flow. Second Edition. McGraw-Hill. New York, 1991.

[6] J. Ferre-Gine, R. Rallo, A. Arenas and F. Giralt, Extraction of Structures from Turbulent Signals, Artificial Intelligence in Engineering, Vol 11, issue 4, pp. 413 - 419. Oct. 1997

[7] R. Mañe, On the Dimension of the Compact Invariant Sets of Certain Non-Linear Maps, Dynamical Systems and Turbulence, "Warwick 1980 Lecture Notes in Mathematics”, Vol. 898, pp. 230, D. Rand and L.S. Young (Eds.), Springer Verlag, 1981.

[8] F. Takens, On the Numerical Determination of the Dimension of an Attractor, Dynamical Systems and Turbulence, “Warwick 1980 Lecture Notes in Mathematics”, V. 898, pp.366-381, D. Rand and L.S. Young (Eds.), Springer Verlag, 1981.

[9] R.J. Duro and J. Santos, Discrete Time Backpropagation for Training Synaptic Delay Based Artificial Neural Networks, IEEE Transactions on Neural Networks, Vol.10, No. 4, pp. 779-789, 1999.

[10] F. López Peña, A. Barro Eiroa and R.J. Duro. Development of a Virtual Instrument for Automatic Calibration of Anemometers. IEEE International Workshop on Virtual and Intelligent Measurement Systems. May, 2001.

[11] S. Haykin and J. Principe, Making Sense of a Complex World, IEEE Signal Processing Magazine, pp. 66-81, May 1998.

[12] H.D.I. Abarbanel, Analysis of Observed Chaotic Data, Springer Verlag, 1986.

[13] H.D.I. Abarbanel, Local False Nearest Neighbours and Dynamical Dimensions from Observed Chaotic Data, Phys Rev. E., Vol. 47, pp. 3057-3068, 1993.

[14] A.M. Fraser, Information and Entropy in Strange Attractors, IEEE Trans. Inf. Theory, Vol. 35, pp. 245-262, 1989.

[15] J. Santos and R.J. Duro, Pi-DTB Discrete Time Backpropagation with Product Units, Connectionist Models of Neurons, Learning Processes and Artificial Intelligence, Springer Verlag, pp 207-214, 2001. 